PROCEEDINGS OF THE

AMERICAN MATHEMATICAL SOCIETY

Volume 125, Number 2, February 1997, Pages 485-492

S 0002-9939(97)03712-X

\title{
A NOTE ON THE BOGOMOLOV-TYPE SMOOTHNESS ON DEFORMATIONS OF THE REGULAR PARTS OF ISOLATED SINGULARITIES
}

\author{
KIMIO MIYAJIMA
}

(Communicated by Peter Li)

\begin{abstract}
We apply the Tian-Todorov method, proving the Bogomolov smoothness theorem (for deformations of compact Kähler manifolds) to deformations of the regular part of a Stein space with a finite number of isolated singular points. By the argument based on the Hodge structure on a strongly pseudo-convex Kähler domain or on a punctured Kähler space, we obtain an unobstructed subspace of the infinitesimal deformation space.
\end{abstract}

\section{INTRODUCTION}

In this paper, we will consider Bogomolov-type smoothness on deformations of the regular part of a normal isolated singularity. In [B], F. Bogomolov showed the following new smoothness theorem for the versal family of $n$-dimensional compact Kähler manifold: If there exists a non-vanishing holomorphic (n,0)-form, or if there exists a non-degenerate holomorphic (2,0)-form and $b_{1}=0$, then the versal deformation space of that Kähler manifold is smooth. G. Tian and A. Todorov rephrased it in order to show the smoothness of the moduli space of compact CalabiYau manifolds ([Ti], [To]). Our aim is to consider smoothness of this type in the case of deformations of a normal isolated singularity. Since the smoothness of the versal family is a problem of formal deformation theory and the formal deformation theory of an isolated singularity is equivalent to that of its regular part if the depth of the singularity is greater than or equal to 3 (cf. [S]), we will consider formal deformations of the regular part of an isolated singularity. Let $V$ be a normal Stein space with a finite number of isolated singular points $S:=\left\{p_{1}, \ldots, p_{k}\right\}, \pi: X \rightarrow V$ a resolution of singularities, and denote $E:=\pi^{-1}(S)$. Then $X$ is a strongly pseudoconvex Kähler manifold with an exceptional subset $E$. Let $r: X \rightarrow[-\infty,+\infty)$ be a strictly plurisubharmonic exhaustion function such that $E=\bigcap_{\epsilon} \Omega_{\epsilon}$, where $\Omega_{\epsilon}:=\{x \in X \mid r(x)<\epsilon\}$. We may assume that $d r \neq 0$ on $\Omega_{a} \backslash E$ and outside $\Omega_{b}$ for some $a \leq b$.

We consider deformations of $U:=X \backslash E$. As was shown in [Ak-M], even if $K_{U}$ is trivial, the (formal) versal family of deformations of $U$ is not necessarily smooth. In fact, there might be an obstructed infinitesimal deformation class in $H^{1}\left(U, \Theta_{U}\right)$.

Received by the editors August 14, 1995.

1991 Mathematics Subject Classification. Primary 32G05; Secondary 14B07, 13D10.

Key words and phrases. Deformation, isolated singularity, Hodge structure.

Partially supported by The Sumitomo Foundation. 
So, in this paper, we will consider unobstructedness of a subspace of $H^{1}\left(U, \Theta_{U}\right)$, where we call a subspace unobstructed if there exists a formal smooth family whose infinitesimal deformation space coincides with that subspace.

The main reason for the failure of the analogue of the Bogomolov smoothness theorem in the case of deformation of $U$ is the non-existence of pure Hodge structure on $H^{*}(U, \mathbf{C})$. However, since there exists a pure Hodge structure of weight $\geq n+1$ on $H^{*}\left(\Omega_{c}, \mathbf{C}\right), n=\operatorname{dim} V$ (cf. [D], [O1], [O-T]), we can expect some effect on deformations of $U$. The following is the main theorem.

Theorem A. Suppose that there exists an effective divisor $D \in\left|-K_{X}\right|$ with $S u p p D \subset E$ or $K_{X}$ is trivial. Then the subspace

$$
\operatorname{Im}\left(H^{1}\left(X, \Theta_{X}(-D)\right) \rightarrow H^{1}\left(U, \Theta_{U}\right)\right)
$$

is unobstructed, where we denote $\Theta_{X}(-D)=\Theta_{X}$ if $K_{X}=1_{X}$.

Since any relatively compact Stein domain of $V$ can be completed to a projective variety (cf. $[\mathrm{Ar}],[\mathrm{L}])$, we may assume that $X$ is an open domain of a compact Kähler manifold $\bar{X}$. Using the pure Hodge structure of weight $\leq n-2$ on the punctured Kähler space $X^{\prime}:=\bar{X} \backslash E$ (cf. [O2]), we also have

Theorem B. Suppose $\operatorname{dim} V \geq 4$ and there exists a non-degenerate holomorphic $(2,0)$-form $\omega$ on $U$ which is extendable to a non-degenerate holomorphic $(2,0)$-form over $X^{\prime}$ or to a closed meromorphic $(2,0)$-form over $X^{\prime}$ such that the component of the local expression $\left(\omega_{i j}\right)$ of that meromorphic (2,0)-form has a holomorphic inverse. Then the subspace $\operatorname{Im}\left(H^{1}\left(X^{\prime}, \Omega_{X^{\prime}}^{1}\right) \rightarrow H^{1}\left(U, \Omega_{U}^{1}\right) \simeq H^{1}\left(U, \Theta_{U}\right)\right)$ is unobstructed.

We will prove these theorems in $\S \S 2$ and 3 . Our proof is an analogue of the TianTodorov argument and depends on the Hodge structures stated above. Hence, in $\S 1$, we will derive the key properties for that argument from the Hodge structure on $\Omega_{c}$ or on $X^{\prime}$ (Corollary 1.4). In $\S 4$, we will mention briefly an example discussed in $[\mathrm{M}]$.

I would like to thank Professors T. Ohsawa and M. Tomari for helpful discussions in this work. In particular, the proof of Proposition 1.3 is due to Professor T. Ohsawa.

$$
\S 1 . \partial \bar{\partial} \text {-LEMMA AND } I_{X}^{n-1,1}=H_{X}^{n-1,1}
$$

Let $X$ be a complex manifold with $\operatorname{dim}_{\mathbf{C}} X=n$. Consider the double complex $\left(A_{X}^{p, q}, \partial, \bar{\partial}\right)$. The Tian-Todorov method heavily depends on the Hodge structure about this double complex, in particular on the following two properties:

(1.1) $I_{X}^{n-1,1}=H_{X}^{n-1,1}$.

(1.2) $\left(\partial \bar{\partial}\right.$-lemma at $\left.A_{X}^{n-1,2}\right)$ If an $(n-1,2)$-form is $d$-closed and $\partial$-exact, then it is $\partial \bar{\partial}$-exact.

Here we denote by $H_{X}^{p, q}$ the $q$-th Dolbeault cohomology group of $\left(A_{X}^{p, \bullet}, \bar{\partial}\right)$, and $I_{X}^{p, q}:=\operatorname{Ker} \bar{\partial} \cap Z_{X}^{p, q} / \bar{\partial} A_{X}^{p, q-1} \cap Z_{X}^{p, q}$ with $Z_{X}^{p, q}:=\left\{\alpha \in A_{X}^{p, q} \mid \partial \alpha=0\right\}$.

In this section, we will discuss the property $I_{X}^{p, q}=H_{X}^{p, q}$ and the $\partial \bar{\partial}$-lemma on a strongly pseudo-convex Kähler domain $\Omega_{c}$ or on a punctured Kähler space $X^{\prime}$. The Hodge structure on $\Omega_{c}$ (resp. on $X^{\prime}$ ), established in [O1] (see also [D], [O-T]) (resp. in [O2]), asserts the existence of $\varphi_{\nu}^{p, q} \in A_{X}^{p, q}\left(p+q=k, 1 \leq \nu \leq h_{X}^{p, q}<+\infty\right)$ satisfying the following $\left(k \geq n+1\right.$ for $\Omega_{c}$ and $k \leq n-2$ for $\left.X^{\prime}\right)$ :

(1.3) $d \varphi_{\nu}^{p, q}=0$ 


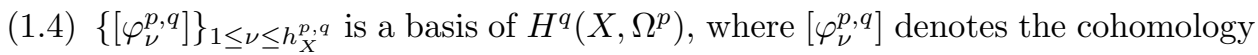
class of $\varphi_{\nu}^{p, q}$ in $H^{q}\left(X, \Omega^{p}\right)$,

(1.5) $\left\{\left[\varphi_{\nu}^{p, q}\right]\right\}_{p+q=k, 1 \leq \nu \leq h_{X}^{p, q}}$ is a basis of $H^{k}(X, \mathbf{C})$, where $\left[\varphi_{\nu}^{p, q}\right]$ denotes the cohomology class of $\varphi_{\nu}^{p}, q$ in $H^{k}(X, \mathbf{C})$,

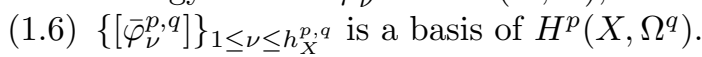

Remark. The condition (1.6) is superfluous; in fact, it follows from (1.3)-(1.5).

Lemma 1.1. Let $k \geq 1$ and suppose that there exist $\varphi_{\nu}^{p, q} \in A_{X}^{p, q}\left(1 \leq \nu \leq h_{X}^{p, q}\right)$ satisfying (1.3)-(1.6) for all $(p, q)$ with $p+q=k$. If $\alpha^{p, q} \in A_{X}^{p, q}(p+q=k)$ is $\bar{\partial}$-closed and $\partial$-exact, then $\alpha^{p, q} \in \bigoplus_{p^{\prime}+q^{\prime}=k-1, q^{\prime} \geq q} d A_{X}^{p^{\prime}, q^{\prime}}$.

Proof. Throughout the proof, we use the notation $\varphi^{s, t}$ as a linear combination of $\left\{\varphi_{\nu}^{s, t}\right\}_{1 \leq \nu \leq h_{X}^{s, t}}$ and $y^{s, t}$ as an element of $A_{X}^{s, t}$. Note that $A_{X}^{s, t}=0$ unless $s(s-n) \leq 0$ and $t(t-n) \leq 0$. Let $\alpha^{p, q}=\partial y^{p-1, q}$. Since $\bar{\partial} \alpha^{p, q}=0$, we have $\partial y^{p-1, q}=$ $\varphi^{p, q}-\bar{\partial} y^{p, q-1}$ by (1.4). Since $\bar{\partial} \partial y^{p, q-1}=0$, we have $\partial y^{p, q-1}=\varphi^{p+1, q-1}-\bar{\partial} y^{p+1, q-2}$ by (1.2) again. Repeating this argument, we have

$$
\begin{gathered}
\partial y^{p-1, q}+d\left(y^{p, q-1}+y^{p+1, q-2}+\cdots+y^{n, p+q-n-1}\right) \\
=\varphi^{p, q}+\varphi^{p+1, q-1}+\cdots+\varphi^{n, p+q-n} .
\end{gathered}
$$

On the other hand, applying the above argument to $\partial \overline{y^{p-1, q}}$, we have

$$
\begin{gathered}
\bar{\partial} y^{p-1, q}+d\left(y^{p-2, q+1}+y^{p-3, q+2}+\cdots+y^{p+q-n-1, n}\right) \\
=\varphi^{p-1, q+1}+\varphi^{p-2, q+2}+\cdots+\varphi^{p+q-n, n} .
\end{gathered}
$$

Hence we have $\left[\varphi^{p+q-n, n}+\cdots+\varphi^{p, q}+\cdots+\varphi^{n, p+q-n}\right]=0$ in $H^{k}(X, \mathbf{C})$. By (1.5), we have $\varphi^{p-1, q+1}=\varphi^{p-2, q+2}=\cdots=\varphi^{p+q-n, n}=0$ and

$$
\alpha^{p, q}=d\left(y^{p-1, q}+y^{p-2, q+1}+\cdots+y^{p+q-n-1, n}\right) .
$$

Proposition 1.2. Under the assumption of Lemma 1.1, the $\partial \bar{\partial}$-lemma holds at $A_{X}^{p, q}$ for all $(p, q)$ with $p+q=k$ and $r \leq q \leq n$ if and only if $I_{X}^{s, t}=H_{X}^{s, t}$ holds for all $(s, t)$ with $s+t=k-1$ and $r \leq t \leq n$.

Proof. We use the same notation as in the proof of Lemma 1.1. Let $\alpha^{p, q} \in A_{X}^{p, q}$ and $\beta^{p-1, q} \in A_{X}^{p-1, q}(p+q=k)$ satisfy $\bar{\partial} \alpha^{p, q}=0$ and $\alpha^{p, q}=\partial \beta^{p-1, q}$. Then by Lemma 1.1,

$$
\alpha^{p, q}=d\left(y^{p-1, q}+y^{p-2, q+1}+\cdots+y^{p+q-n-1, n}\right) .
$$

Since $I_{X}^{p+q-n-1, n}=H_{X}^{p+q-n-1, n}$ holds, we have

$$
y^{p+q-n-1, n}=\varphi^{p+q-n-1, n}+\bar{\partial} \gamma^{p+q-n-1, n-1} .
$$

Since $d \varphi^{p+q-n-1, n}=0$, if we set

$$
y^{\prime p+q-n-2, n-1}:=y^{p+q-n-2, n-1}-\partial \gamma^{p+q-n-1, n-1},
$$

we have $\bar{\partial} y^{\prime p+q-n-2, n-1}=0$ and $\partial y^{\prime p+q-n-2, n-1}=\partial y^{p+q-n-2, n-1}$. Repeating this argument, we have a $\gamma^{p-1, q-1} \in A_{X}^{p-1, q-1}$ such that

$$
\partial\left(y^{p+q-n-2, n-1}-\bar{\partial} \gamma^{p-1, q-1}\right)=0 .
$$

Therefore we have $\alpha^{p, q}=-\partial \bar{\partial} \gamma^{p-1, q-1}$. The only if part is proved by a standard argument. 
About $I_{\Omega_{c}}^{s, t}=H_{\Omega_{c}}^{s, t}$ for $s+t=n$, we have

Proposition 1.3. $I_{\Omega_{c}}^{s, t}=H_{\Omega_{c}}^{s, t}$ holds for $(s, t)$ with $s+t=n$ and $t \geq 1$.

Proof (due to T.Ohsawa). We use the same notation as in the proof of Lemma 1.1. We introduce a complete Kähler metric over $\Omega_{c}$ associated to the Kähler form $\omega-\sqrt{-1} \partial \bar{\partial} \log (c-r)$, where $\omega$ denotes the Kähler form on $X$. Then it is proved in [O1] that the following hold:

(i) For $k \geq n+1$, the restriction $\beta_{\mid \Omega_{c}}$ of a $\beta \in L_{l o c}^{k}\left(\Omega_{c+\epsilon}\right)(\epsilon>0)$ is an $L^{2}$-form on $\Omega_{c}$.

(ii) For $k \geq n+1$, the natural homomorphism $H^{k}\left(\Omega_{c}, \mathbf{C}\right) \rightarrow H_{(2)}^{k}\left(\Omega_{c}, \mathbf{C}\right)$ is an isomorphism, where $H_{(2)}^{k}\left(\Omega_{c}, \mathbf{C}\right)$ denotes the $L^{2}$-cohomology group.

(iii) The image of $\bar{\partial}: L_{\Omega_{c}}^{p, q} \rightarrow L_{\Omega_{c}}^{p, q+1}$ is closed, where $L_{\Omega_{c}}^{p, q}$ denotes the space of $L^{2}$ $(p, q)$-forms on $\Omega_{c}$.

Let $\alpha^{p, q} \in A_{\Omega_{c}}^{p, q}(p+q=n, q \geq 1)$ be a $\bar{\partial}$-closed form. It is enough to find a $\gamma^{p, q-1} \in A_{\Omega_{c}}^{p, q-1}$ satisfying $\partial \bar{\partial} \gamma^{p, q-1}=\partial \alpha^{p, q}$. Since $H^{q}\left(X, \Omega^{p}\right) \rightarrow H^{q}\left(\Omega_{c}, \Omega^{p}\right)$ is an isomorphism, we may assume that $\alpha^{p, q}$ can be extendable to $X$. By (i) and (ii), we have that $\partial \alpha^{p, q}$ is an $L^{2}$-form, and $\partial \alpha^{p, q}=d y$ for some $C^{\infty}$ and $L^{2}$-form $y$ on $\Omega_{c}$. Let $y=y^{0, n}+y^{1, n-1}+\cdots+y^{n, 0}, y^{s, t} \in A_{\Omega_{c}}^{s, t} \cap L_{\Omega_{c}}^{s, t}$. By (iii), we can find $\gamma^{0, n-1} \in$ $A_{\Omega_{c}}^{0, n-1} \cap L_{\Omega_{c}}^{0, n-1}$ such that $\bar{\partial}^{*}\left(y^{0, n}-\bar{\partial} \gamma^{0, n-1}\right)=0$ and $\bar{\partial}^{*} \gamma^{0, n-1}=0$ hold. Hence we have $\square\left(y^{0, n}-\bar{\partial} \gamma^{0, n-1}\right)=0$. This implies $\partial\left(y^{0, n}-\bar{\partial} \gamma^{0, n-1}\right)=0$, since $\square=\frac{1}{2} \Delta$. If we set $y^{\prime 1, n-1}:=y^{1, n-1}-\partial \gamma^{0, n-1} \in A_{\Omega_{c}}^{1, n-1} \cap L_{\Omega_{c}}^{1, n-1}$, we have $\bar{\partial} y^{\prime 1, n-1}=0$ and $\partial y^{1, n-1}=\partial y^{1, n-1}$. Here we remark that $\partial \gamma^{0, n-1} \in L_{\Omega_{c}}^{1, n-1}$ holds, since $\gamma^{0, n-1}$, $\bar{\partial} \gamma^{0, n-1}$ and $\bar{\partial}^{*} \gamma^{0, n-1}$ are all $L^{2}$-forms. Repeating this argument, we finally have a $\gamma_{1}^{p, q-1} \in A_{\Omega_{c}}^{p, q-1} \cap L_{\Omega_{c}}^{p, q-1}$ such that $\partial y^{p, q}=\partial \bar{\partial} \gamma_{1}^{p, q-1}$ holds. Next, by applying the same argument to $\overline{y^{n, 0}}, \ldots, \overline{y^{p+1, q-1}}$, we have a $\gamma_{2}^{p, q-1} \in A_{\Omega_{c}}^{p, q-1} \cap L_{\Omega_{c}}^{p, q-1}$ such that $\bar{\partial} y^{p+1, q-1}=\partial \bar{\partial} \gamma_{2}^{p, q-1}$ holds. Therefore, we have

$$
\partial \bar{\partial}\left(\gamma_{1}^{p, q-1}-\gamma_{2}^{p, q-1}\right)=\partial y^{p, q}+\bar{\partial} y^{p+1, q-1}=\partial \alpha^{p, q} .
$$

Therefore we have

Corollary 1.4. (1) $I_{\Omega_{c}}^{p, q}=H_{\Omega_{c}}^{p, q}$ holds if $p+q \geq n+1$, or if $p+q=n$ and $q \geq 1$. The $\partial \bar{\partial}$-lemma holds at $A_{\Omega_{c}}^{p, q}$ if $p+q \geq n+1$,

(2) $I_{X^{\prime}}^{p, q}=H_{X^{\prime}}^{p, q}$ holds if $p+q \leq n-2$. The $\partial \bar{\partial}$-lemma holds at $A_{X^{\prime}}^{p, q}$ if $p+q \leq n-2$.

\section{§2. Proof of Theorem A}

In this section, we consider deformations of $\Omega_{c}$ and their effect on deformations of $U:=\Omega_{c} \backslash E$. We remark that, since the (formal) deformation theories of $\Omega_{c}$ and $\Omega_{c} \backslash E$ are both independent of $c \in(-\infty, a) \cup[b, \infty)$ and we consider $V$ as a germ of complex space at $S$, we may consider $\Omega_{c}$ instead of $X$; hence we may assume that Corlllary 1.4 (1) holds on $X$. Then, the following analogue of the Bogomolov smoothness theorem is clear.

Theorem 2.1. If $K_{X}$ is trivial, then the space $H^{1}\left(X, \Theta_{X}\right)$ is unobstructed for deformations of $X$. 
Theorem A for the case of $K_{X}=1_{X}$ immediately follows from Theorem 2.1. Next, we consider the situation that there exists a meromorphic $(n, 0)$-form $\omega$ with only poles $D$ supported in $E$, and prove the following theorem:

Theorem 2.2. Suppose that there exists an effective divisor $D \in\left|-K_{X}\right|$ with $S u p p D \subset E$. Then the subspace $\operatorname{Im}\left(H^{1}\left(X, \Theta_{X}(-D)\right) \rightarrow H^{1}\left(U, \Theta_{U}\right)\right)$ is unobstructed.

Proof. Since $\iota(\phi)=\omega\rfloor \phi$, we have an isomorphism

$$
\iota: A_{X}^{0, q}\left(T^{\prime} X(-D)\right) \longrightarrow A_{X}^{n-1, q} .
$$

Then by direct calculations, we have

(2.1) If $\phi \in A_{X}^{0,1}\left(T^{\prime} X(-D)\right)$, then $\bar{\partial} \phi \in A_{X}^{0,2}\left(T^{\prime} X(-D)\right)$.

(2.2) If $\phi, \psi \in A_{X}^{0,1}\left(T^{\prime} X(-D)\right)$, then $[\phi, \psi] \in A_{X}^{0,2}\left(T^{\prime} X(-D)\right)$.

And by the same calculations as in [Ti] or [To], we have

(2.3) $\bar{\partial} \iota=\iota \bar{\partial}$.

(2.4) If $\partial \alpha=\partial \beta=0$, then $\left.\iota\left[\iota^{-1} \alpha, \iota^{-1} \beta\right]=\partial\left(\iota^{-1} \alpha\right\rfloor \beta\right)$.

By (2.3), we have an isomorphism $H^{q}\left(X, \Theta_{X}(-D)\right) \cong H^{q}\left(X, \Omega_{X}^{n-1}\right)$. By Corollary 1.4 (1), we can choose $\left\{\alpha_{\nu}^{n-1,1}\right\}_{1 \leq \nu \leq d} \subset Z_{X}^{n-1,1}$ such that $\left\{\left[\iota^{-1} \alpha_{\nu \mid U}^{n-1,1}\right]\right\}_{1 \leq \nu \leq d}$ is a basis of $\operatorname{Im}\left(H^{1}\left(X, \Theta_{X}(-D)\right) \rightarrow H^{1}\left(U, \Theta_{U}\right)\right)$. Then, by the same argument of [Ti] or [To], we can construct a formal power series $\alpha(s) \in A_{X}^{n-1,1}\left[\left[s_{1}, \ldots, s_{d}\right]\right]$ satisfying

(2.5) $\left.\bar{\partial} \alpha(s)-\frac{1}{2} \partial\left(\iota^{-1} \alpha(s)\right\rfloor \alpha(s)\right)=0$,

(2.6) $\alpha(s) \equiv \sum_{\nu=1}^{d} \alpha_{\nu}^{n-1,1} s_{\nu} \bmod \mathfrak{m}^{2}$, where $\mathfrak{m}$ denotes the maximal ideal of $\mathbf{C}\left[\left[s_{1}, \ldots, s_{d}\right]\right]$.

Hence if we set $\phi(s):=\iota^{-1} \alpha(s)$, then it satisfies

(2.7) $\bar{\partial} \phi(s)-\frac{1}{2}[\phi(s), \phi(s)]=0$,

(2.8) $\phi(s) \equiv \sum_{\nu=1}^{d} \iota^{-1} \alpha_{\nu}^{n-1,1} s_{\nu} \bmod \mathfrak{m}^{2}$.

By restricting $\phi(s)$ on $U$, we have a formal family of deformations of $U$ whose infinitesimal deformation space is $\operatorname{Im}\left(H^{1}\left(X, \Theta_{X}(-D)\right) \rightarrow H^{1}\left(U, \Theta_{U}\right)\right)$. Hence that space is unobstructed.

This completes the proof of Theorem 2.2.

\section{§3. Proof of Theorem B}

In this section, we consider deformations of $X^{\prime}$ and their effect on deformations of $U$. By Corollary $1.4(2)$, the following analogue of the Bogomolov smoothness theorem is clear. (Note that all holomorphic $(2,0)$-forms on $X^{\prime}$ are $d$-closed by Corollary $1.4(2)$.)

Theorem 3.1. If there exists a non-degenerate holomorphic $(2,0)$-form on $X^{\prime}$, then the space $H^{1}\left(X^{\prime}, \Theta_{X^{\prime}}\right)$ is unobstructed for deformations of $X^{\prime}$.

Next, we suppose that there exists a non-degenerate holomorphic $(2,0)$-form $\omega$ on $U$ which is extendable to a closed meromorphic $(2,0)$-form on $X^{\prime}$ such that $\left(\omega_{i j}\right)$ has a holomorphic inverse, where we denote the meromorphic $(2,0)$-form by $\omega=\frac{1}{2} \sum_{i, j} \omega_{i j} d z^{i} \wedge d z^{j}$, with local meromorphic functions $\omega_{i j}$, with respect to a local coordinate $\left(z^{1}, \ldots, z^{n}\right)$ of $X^{\prime}$. Then we have 
Theorem 3.2. Suppose that there exists a non-degenerate holomorphic $(2,0)$-form $\omega$ on $U$ as above. Then the subspace $\operatorname{Im}\left(H^{1}\left(X^{\prime}, \Omega_{X^{\prime}}^{1}\right) \rightarrow H^{1}\left(U, \Omega_{U}^{1}\right) \simeq H^{1}\left(U, \Theta_{U}\right)\right)$ is unobstructed.

Proof. Since $\iota(\phi)=\omega\rfloor \phi$, we have homomorphisms

$$
\iota^{-1}: A_{X^{\prime}}^{1, q} \longrightarrow A_{X^{\prime}}^{0, q}\left(T^{\prime} X^{\prime}\right)
$$

and

$$
\iota: \iota^{-1}\left(A_{X^{\prime}}^{1, q}\right) \longrightarrow A_{X^{\prime}}^{1, q}
$$

Lemma 3.3. (1) $\bar{\partial} \iota^{-1}=\iota^{-1} \bar{\partial}$.

(2) If $\partial \xi=\partial \eta=0$, then $\left.\iota\left[\iota^{-1} \xi, \iota^{-1} \eta\right]=\partial\left(\iota^{-1} \xi\right\rfloor \eta\right)$. In particular, $\left[\iota^{-1} \xi, \iota^{-1} \eta\right] \in$ $\iota^{-1}\left(A_{X^{\prime}}^{1,2}\right)$.

Proof. (1) is clear.

(2) Let $\left(h^{\alpha, i}\right)$ be a holomorphic $(n, n)$-matrix satisfying $\left(h^{\alpha, i}\right)\left(\omega_{i, \gamma}\right)=\left(\delta_{\gamma}^{\alpha}\right)$ and $\left(\omega_{i, \gamma}\right)\left(h^{\gamma, j}\right)=\left(\delta_{i}^{j}\right)$. Suppose $\xi=\sum_{i, \beta} \xi_{i, \bar{\beta}} d z^{i} \wedge d \bar{z}^{\beta}$ and $\eta=\sum_{j, \delta} \eta_{j, \bar{\delta}} d z^{j} \wedge$ $d \bar{z}^{\delta}$ satisfy $\partial \xi=\partial \eta=0$. Since

$$
\begin{aligned}
{\left[\iota^{-1} \xi, \iota^{-1} \eta\right]=\frac{1}{2} \sum_{\alpha, \beta, \delta} } & \sum_{i, j, \gamma}\left\{\left(h^{\gamma i} h^{\alpha j} \xi_{i \bar{\beta}} \frac{\partial \eta_{j} \bar{\delta}}{\partial z^{\gamma}}-h^{\alpha i} h^{\gamma j} \eta_{j \bar{\delta}} \frac{\partial \xi_{i \bar{\beta}}}{\partial z^{\gamma}}\right)\right. \\
& -\left(h^{\gamma i} h^{\alpha j} \xi_{i \bar{\delta}} \frac{\partial \eta_{j \bar{\beta}}}{\partial z^{\gamma}}-h^{\alpha i} h^{\gamma j} \eta_{j \bar{\beta}} \frac{\partial \xi_{i \bar{\delta}}}{\partial z^{\gamma}}\right) \\
+ & \left.\left(h^{\gamma i} \frac{\partial h^{\alpha j}}{\partial z^{\gamma}}-h^{\gamma j} \frac{\partial h^{\alpha i}}{\partial z^{\gamma}}\right)\left(\xi_{i \bar{\beta}} \eta_{j \bar{\delta}}-\xi_{i \bar{\delta}} \eta_{j \bar{\beta}}\right)\right\} \frac{\partial}{\partial z^{\alpha}} \otimes d \bar{z}^{\beta} \wedge d \bar{z}^{\delta}
\end{aligned}
$$

and $\partial \xi=\partial \eta=0$, we have

$$
\begin{aligned}
\iota\left[\iota^{-1} \xi, \iota^{-1} \eta\right]= & \frac{1}{2} \sum_{k, \beta, \delta} \sum_{i, j, \alpha, \gamma}\left\{h^{\gamma i}\left(\xi_{i \bar{\beta}} \frac{\partial \eta_{\gamma \bar{\delta}}}{\partial z^{k}}-\xi_{i \bar{\delta}} \frac{\partial \eta_{\gamma \bar{\beta}}}{\partial z^{k}}\right)-h^{\gamma j}\left(\frac{\partial \xi_{\gamma \bar{\beta}}}{\partial z^{k}} \eta_{j \bar{\delta}}-\frac{\partial \xi_{\gamma \bar{\delta}}}{\partial z^{k}} \eta_{j \bar{\beta}}\right)\right. \\
& \left.+\omega_{k \alpha}\left(h^{\gamma i} \frac{\partial h^{\alpha j}}{\partial z^{\gamma}}-h^{\gamma j} \frac{\partial h^{\alpha i}}{\partial z^{\gamma}}\right)\left(\xi_{i \bar{\beta}} \eta_{j \bar{\delta}}-\xi_{i \bar{\delta}} \eta_{j \bar{\beta}}\right)\right\} d z^{k} \wedge d \bar{z}^{\beta} \wedge d \bar{z}^{\delta} .
\end{aligned}
$$

On the other hand, since $\left.\iota^{-1} \xi\right\rfloor \eta=\frac{1}{2} \sum_{\beta, \delta} \sum_{i, \alpha} h^{\alpha i}\left(\xi_{i \bar{\beta}} \eta_{\alpha \bar{\delta}}-\xi_{i \bar{\delta}} \eta_{\alpha \bar{\beta}}\right) d \bar{z}^{\beta} \wedge d \bar{z}^{\delta}$ and $h^{i \alpha}=-h^{\alpha i}$, we have

$$
\begin{aligned}
\left.\partial\left(\iota^{-1} \xi\right\rfloor \eta\right)= & \frac{1}{2} \sum_{k, \beta, \delta} \sum_{i, \alpha}\left\{h^{\alpha i}\left(\xi_{i \bar{\beta}} \frac{\partial \eta_{\alpha \bar{\delta}}}{\partial z^{k}}-\xi_{i \bar{\delta}} \frac{\partial \eta_{\alpha \bar{\beta}}}{\partial z^{k}}\right)\right. \\
& \left.-h^{i \alpha}\left(\frac{\partial \xi_{i \bar{\beta}}}{\partial z^{k}} \eta_{\alpha \bar{\delta}}-\frac{\partial \xi_{i \bar{\delta}}}{\partial z^{k}} \eta_{\alpha \bar{\beta}}\right)+\frac{\partial h^{\alpha i}}{\partial z^{k}}\left(\xi_{i \bar{\beta}} \eta_{\alpha \bar{\delta}}-\xi_{i \bar{\delta}} \eta_{\alpha \bar{\beta}}\right)\right\} d z^{k} \wedge d \bar{z}^{\beta} \wedge d \bar{z}^{\delta} .
\end{aligned}
$$

Hence (2) follows from the following

\section{Claim.}

$$
\frac{\partial h^{j i}}{\partial z^{k}}=\sum_{\alpha, \gamma} \omega_{k \alpha}\left(h^{\gamma i} \frac{\partial h^{\alpha j}}{\partial z^{\gamma}}-h^{\gamma j} \frac{\partial h^{\alpha i}}{\partial z^{\gamma}}\right)
$$


Proof. By $h^{j i}=\sum_{\alpha, \gamma} h^{j \gamma} \omega_{\gamma \alpha} h^{\alpha i}$ and $d \omega=0$, we have

$$
\frac{\partial h^{j i}}{\partial z^{k}}=-\sum_{\alpha, \gamma} h^{j \gamma}\left(\frac{\partial \omega_{k \alpha}}{\partial z^{\gamma}}+\frac{\partial \omega_{\gamma k}}{\partial z^{\alpha}}\right) h^{\alpha i} .
$$

Hence the claim follows from $\sum_{\alpha} \omega_{k \alpha} h^{\alpha i}=\delta_{k}^{i}$ and $\sum_{\gamma} h^{j \gamma} \omega_{\gamma k}=\delta_{k}^{j}$.

Then by the same argument as in $\S 2$, we have a formal power series $\alpha(s) \in$ $A_{X^{\prime}}^{1,1}\left[\left[s_{1}, \ldots, s_{d}\right]\right]$ satisfying

(3.1) $\left.\bar{\partial} \alpha(s)-\frac{1}{2} \partial\left(\iota^{-1} \alpha(s)\right\rfloor \alpha(s)\right)=0$,

(3.2) the linear part of $\iota^{-1} \alpha(s)_{\mid U}$ spans

$$
\operatorname{Im}\left(H^{1}\left(X^{\prime}, \Omega_{X^{\prime}}^{1}\right) \rightarrow H^{1}\left(U, \Omega_{U}^{1}\right) \simeq H^{1}\left(U, \Theta_{U}\right)\right),
$$

where $d=\operatorname{dim}_{\mathbf{C}} \operatorname{Im}\left(H^{1}\left(X^{\prime}, \Omega_{X^{\prime}}^{1}\right) \rightarrow H^{1}\left(U, \Omega_{U}^{1}\right) \simeq H^{1}\left(U, \Theta_{U}\right)\right)$.

Hence, if we set $\phi(s):=\iota^{-1} \alpha(s)_{\mid U}$, then we have

(3.3) $\bar{\partial} \phi(s)-\frac{1}{2}[\phi(s), \phi(s)]=0$,

(3.4) the linear part of $\phi(s)$ spans

$$
\operatorname{Im}\left(H^{1}\left(X^{\prime}, \Omega_{X^{\prime}}^{1}\right) \rightarrow H^{1}\left(U, \Omega_{U}^{1}\right) \simeq H^{1}\left(U, \Theta_{U}\right)\right) .
$$

This completes the proof of Theorem 3.2.

\section{$\S 4$. Examples (Deformations of QuASi-Gorenstein CONe Singularities)}

We consider deformations of quasi-Gorenstein cone singularities as an example of our argument. For detailed discussion, see $[\mathrm{M}]$. Let $Y$ be a projective algebraic manifold and $\pi: F \rightarrow Y$ a negative line bundle. We denote by $X$ the total space of $F$, by $D_{0}$ the 0 -section of $F$ and $U:=X \backslash D_{0}$.

Example 4.1. We suppose that the canonical line bundle $K_{U}$ is trivial. Then $K_{Y} \simeq F^{\mu}$ for some $\mu \in \mathbf{Z}$.

(1) The case of $K_{Y} \simeq F^{\mu}(\mu>1)$ : Since $H^{2}\left(X, \Theta_{X}\right) \simeq H^{2}\left(X, \Omega_{X}^{n-1}\left(K_{X}^{-1}\right)\right)=0$ $([\mathrm{N}])$, the subspace $\operatorname{Im}\left(H^{1}\left(X, \Theta_{X}\right) \rightarrow H^{1}\left(U, \Theta_{U}\right)\right)$ is unobstructed.

(2) The case of $K_{Y} \simeq F^{-\mu}(\mu \geq-1)$ : Since $K_{X} \simeq \pi^{*}\left(F^{-\mu-1}\right)$, by Theorem A, the space $\operatorname{Im}\left(H^{1}\left(X, \Theta_{X}\left(-(\mu+1) D_{0}\right)\right) \rightarrow H^{1}\left(U, \Theta_{U}\right)\right)$ is unobstructed, where we denote $\Theta_{X}\left(-(\mu+1) D_{0}\right):=\Theta_{X}$ if $\mu=-1$.

Example 4.2. Let $Y:=\mathbf{P}\left(T^{*} \mathbf{P}^{n}\right)(n \geq 2)$ and $F$ the dual tautological line bundle. We denote by $\bar{X}$ the associated $\mathbf{P}^{1}$-bundle and by $D_{\infty}$ the $\infty$-section. Then $X:=$ $\bar{X} \backslash D_{\infty}$ is a special case of Example 4.1 (1) and $X^{\prime}:=\bar{X} \backslash D_{0}$ is a punctured Kähler space. Since there exists a closed meromorphic $(2,0)$-form on $X^{\prime}$ which is non-degenerate and holomorphic on $U$ and having a holomorphic inverse on $X^{\prime}$, by Theorem B, the space $\operatorname{Im}\left(H^{1}\left(X^{\prime}, \Omega_{X^{\prime}}^{1}\right) \rightarrow H^{1}\left(U, \Theta_{U}\right)\right)$ is unobstructed.

Remark. In either case of these examples, the above unobstructed subspace of $H^{1}\left(U, \Theta_{U}\right)$ induces an unobstructed subspace of $\operatorname{Ext}^{1}\left(\Omega_{V}^{1}, \mathcal{O}_{V}\right)$, where we denote by $V$ the germ of a normal complex space obtained by contracting $D_{0}$. 


\section{REFERENCES}

[Ak-M] Akahori,T. and Miyajima,K., A note on the analogue of the Bogomolov type theorem on deformations of CR-structures, Canadian Math. Bull. 37 (1994), 8-12. MR 95d:32018

[Ar] Artin,M., Algebraic approximation of structures over complete local rings, Publ. Math. I.H.E.S. 36 (1969), 23-58. MR 42:3087

[B] Bogomolov,F.A., Hamiltonian Kähler manifolds, Dokl. Akad. Nauk. SSSR 243 (1978), 1101-1104; English transl., Soveit Math. Dokl. 19 (1978), 1462-1465. MR 80c:32024

[D] Demailly,J.-P., Cohomology of q-convex spaces in top degrees, Math. Z. 204 (1990), 283-295. MR 91e:32014

[L] Lempert,L., Algebraic approximations in analytic geometry, Invent. Math. 121 (1995), 335354.

[M] Miyajima,K., A Bogomolov-type smoothness on deformations of quasi-Gorenstein cone singularities of dim $\geq 4$, "Geometric Complex Analysis", ed. J. Noguchi et al., World Scientific, 1996, pp. 461-465.

[N] Nakano,S., Vanishing theorems for weakly 1-complete manifolds, II, Publ. R.I.M.S., Kyoto Univ. 10 (1974), 101-110. MR 52:3617

[O1] Ohsawa,T., A reduction theorem for cohomology groups of very strongly q-convex Kähler manifolds, Inv. Math. 63 (1981), 335-354. MR 82k:32032

[O2] _ Hodge spectral sequence on compact Kähler spaces, Publ. R.I.M.S., Kyoto Univ. 23 (1987), 265-274. MR 92f:32050

[O-T] Ohsawa,T. and Takegoshi,K., Hodge spectral sequence on pseudoconvex domains, Math. Z. 197 (1988), 1-12. MR 89d:32042

[S] Schlessinger,M., On rigid singularities, Conference on Complex Analysis, Rice University Studies 59 (1973), no. 1, 147-162. MR 49:9258

[Ti] Tian,C., Smoothness of the universal deformation space of compact Calabi-Yau manifolds and its Petersson-Weil metric, Mathematical Aspects of String Theory , S.T. Yau ed., World Scientific, 1987, pp. 629-646. MR 89m:81001

[To] Todorov,A.N., The Weil-Petersson geometry of the moduli space of $S U(n \geq 3)$ (CalabiYau)Manifolds I, Commun. Math. Phys. 126 (1989), 325-346. MR 91f:32022

Mathematical Institute, College of Liberal Arts, Kagoshima University, KagoshimaSHI 890 , JAPAN

E-mail address: miyajima@cla.kagoshima-u.ac.jp 